\title{
EEG-based approach for recognizing human social emotion perception
}

\author{
Li Zhu a, Chongwei Su b , Jianhai Zhang ${ }^{\text {b }}$, Gaochao Cui c, Andrzej Cichocki b,d,e, Changle Zhou a , \\ Junhua Li f,g,h,i,* \\ ${ }^{a}$ Department of Artificial Intelligence, School of Informatics, Xiamen University, Xiamen 361005, China \\ ${ }^{\mathrm{b}}$ School of Computer Science and Technology, Hangzhou Dianzi University, Hangzhou 310018, China \\ ${ }^{c}$ Rhythm-Based Brain Information Processing Unit, RIKEN, Wako, Saitama 351-0198, Japan \\ ${ }^{\mathrm{d}}$ Skolkovo Institute of Science and Technology (Skoltech), 143026 Moscow, Russia \\ e Department of Informatics, Nicolaus Copernicus University, 87-100 Torun, Poland \\ ${ }^{\mathrm{f}}$ School of Computer Science and Electronic Engineering, University of Essex, Colchester, CO4 3SQ, UK \\ ${ }^{g}$ Laboratory for Brain-Bionic Intelligence and Computational Neuroscience, Wuyi University, Jiangmen, 529020, China \\ ${ }^{\mathrm{h}}$ Singapore Institute for Neurotechnology, National University of Singapore, Singapore, 117456, Singapore

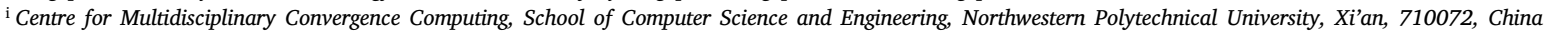

\section{A R T I C L E I N F O}

\section{Keywords:}

Hyper-scanning

EEG

Emotion

Phase lag index

Deep learning

\begin{abstract}
A B S T R A C T
Social emotion perception plays an important role in our daily social interactions and is involved in the treatments for mental disorders. Hyper-scanning technique enables to measure brain activities simultaneously from two or more persons, which was employed in this study to explore social emotion perception. We analyzed the recorded electroencephalogram (EEG) to explore emotion perception in terms of event related potential (ERP) and phase synchronization, and classified emotion categories based on convolutional neural network (CNN). The results showed that (1) ERP was significantly different among four emotion categories (i.e., anger, disgust, neutral, and happy), but there was no significant difference for ERP in the comparison of rating orders (the order of rating actions of the paired participants); (2) the intra-brain phase lag index (PLI) was higher than the inter-brain PLI but its number of connections exhibiting significant difference was less in all typical frequency bands (from delta to gamma); (3) the emotion classification accuracy of inter-PLI-Conv outperformed that of intra-PLI-Conv for all cases of using each frequency band (five frequency bands totally). In particular, the classification accuracies averaged across all participants in the alpha band were $65.55 \%$ and $50.77 \%$ (much higher than the chance level) for the inter-PLI-Conv and intra-PLI-Conv, respectively. According to our results, the emotion category of happiness can be classified with a higher performance compared to the other categories.
\end{abstract}

\section{Introduction}

It is undoubted that emotion is crucial component during humanhuman interaction and human-machine interaction in our everyday life [1]. Emotion is also relevant to some mental diseases and detrimental habits such as internet addiction, tristimania [2], anxiety and social phobia [3]. Although a great deal of efforts have been made by researchers from diverse disciplines (e.g., neuroscience, psychology, and computer science) to investigate emotion perception, the knowledge we currently acquired is still limited [4,5]. Most of the prior studies explored emotion perception in a scenario of one person [6-9], rather than multiple persons who interact each other. In our daily life, people usually perceive emotion during interacting with others [10].
For instance, how you feel yourself and other people during the social interaction $[11,12]$. Therefore, it is better to investigate emotion perception during social interaction.

In recent years, hyper-scanning, a technique for measuring brain activities simultaneously from two or more persons, has been utilized to explore brain-to-brain interactions when two or more persons engage in a task [13]. The hyper-scanning could be categorized as fNIRS (functional near infrared spectroscopy) hyper-scanning [14], fMRI (functional magnetic resonance imaging) hyper-scanning [15] and EEG hyper-scanning [16] according to the signal recorded in the experiment. Because EEG is of high temporal resolution and ease of use with an advantage of low cost [17], we employed EEG hyperscanning in our study. Previous studies revealed that the frontal region was dominantly involved in the emotion recognition, especially facial

\footnotetext{
* Corresponding author at: School of Computer Science and Electronic Engineering, University of Essex, Colchester, CO4 3SQ, UK.

E-mail address: juhalee.bcmi@gmail.com (J. Li).
} 


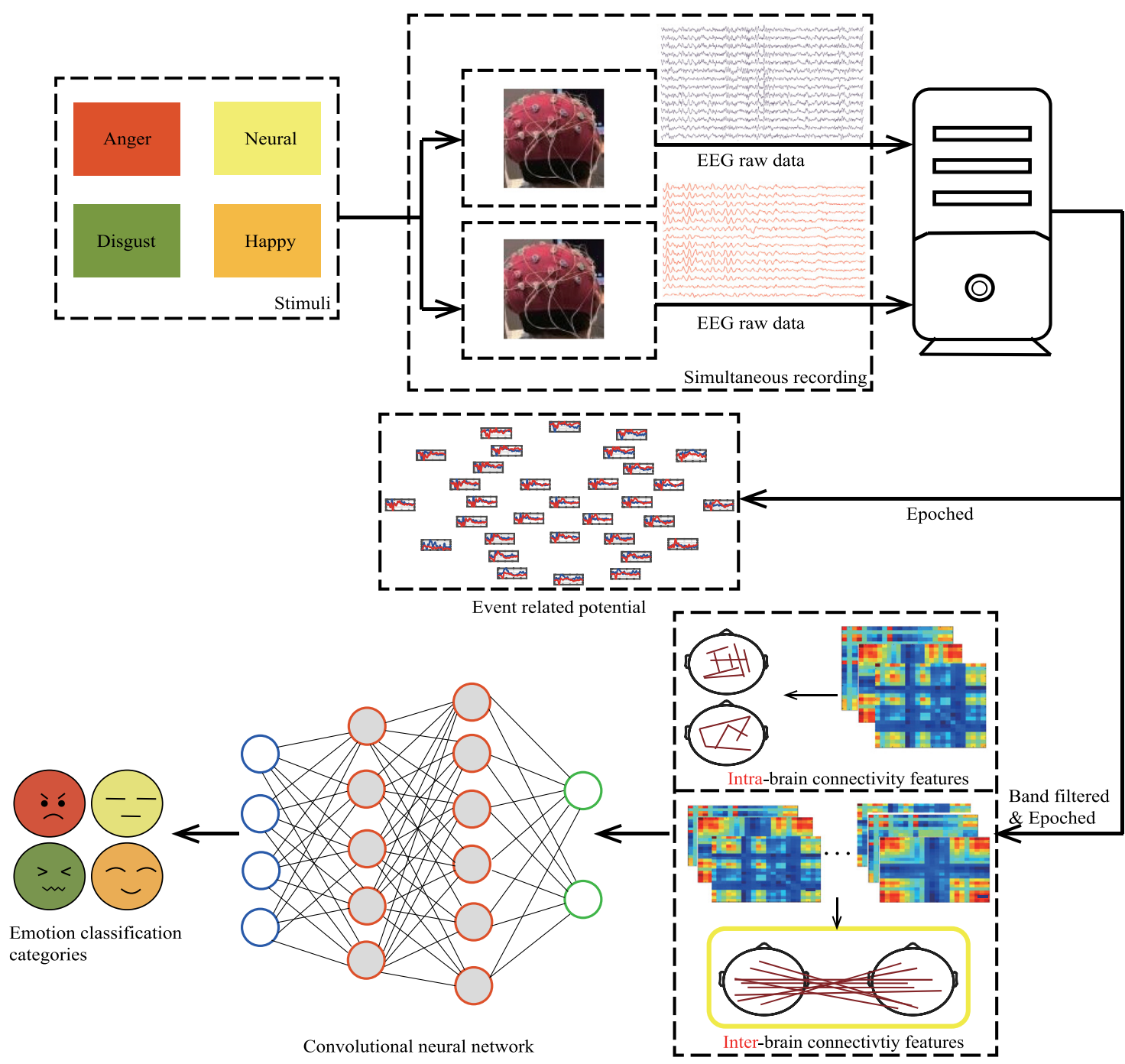

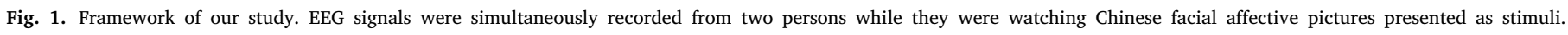

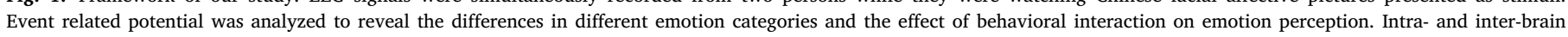

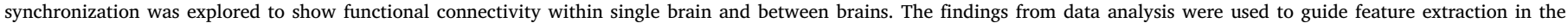
classification. A deep learning model (CNN) was employed to classify emotion categories.

expression recognition [18-20]. This hints us to focus on the frontal region for emotion perception exploration in the context of multiple persons. The concentration on a particular region could also benefit the following step of emotion classification as less amount of data are required for processing.

In this study, we designed an EEG hyper-scanning experiment to explore emotion perception in the scenario of two-person interaction. The characteristics of brain activities were first explored to reveal neural mechanisms related to emotion perception. Then, a deep learning model was utilized to classify different emotion categories based on the findings in the data analysis. The framework of our study was depicted in Fig. 1. The main contributions of this paper are (1) designing an EEG hyper-scanning experiment to explore emotion perception in the scenario of two-person interaction; (2) revealing EEG characteristics associated with emotion perception; (3) evaluating the effectiveness of intra-brain and inter-brain phase synchronization features; (4) recognizing emotions based on EEG signal only using twelve electrodes on the frontal region, which could facilitate the development of portable emotion recognition system. The remainder of the paper is organized as follows. The relevant work is introduced in Section 2, which is followed by the description of experiment design and setup in Section 3. Next, the methodological descriptions of the preprocessing, ERP, synchronization feature extraction, convolutional neural networks are given in Section 4, which is followed by the results in Section 5. Finally, conclusions are drawn in Section 6.

\section{Relevant work}

\subsection{Hyper-scanning experiments}

Hyper-scanning is a technique that enables to measure signals simultaneously from two or more persons. Montague et al. presented an experiment of deception game played by a pair of participants, which is the first fMRI-based hyper-scanning experiment [15]. About ten years later, the first NIRS-based hyper-scanning was reported and NIRS signals were simultaneously collected from two participants engaging in a cooperation task [21]. Babiloni et al. [22] designed the first EEG-based hyper-scanning experiment to investigate the activated regions and the information flow within individual subject and between subjects when they performed a game. In the subsequent years, researchers have achieved more paradigms such as competition and cooperation interactions [16,23-25], motor and verbal communications [26-28]. There have been exploratory studies using hyper-scanning technique to investigate emotional social interactions $[29,30]$. These studies focused on information flow mechanisms between subjects when they have 


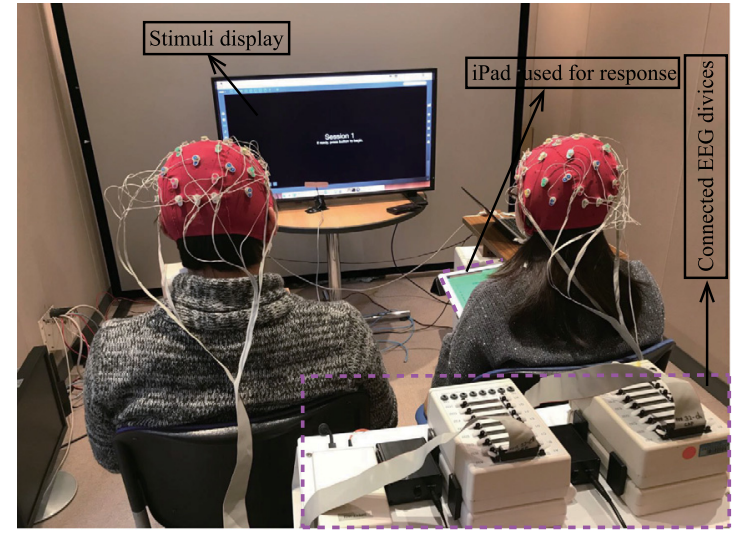

Fig. 2. Experimental environment.

emotional interactions rather than specific emotions and social emotion recognition.

\subsection{EEG-based emotion recognition}

EEG-based emotion recognition has been widely studied based on single-subject experiment. In [31], they used common spatial patterns and support vector machine (SVM) to classify two emotions (i.e., happy and sad) and achieved a classification accuracy of 93.5\%. In [32], SVM classifier was trained using fractal dimension features and utilized to classify three emotions (i.e., anger, happy, and calm). The average classification accuracy was $60 \%$. In [33], an unsupervised EEG feature extraction using auto-encoder method was proposed and the classification accuracy for three emotions ranged from $44 \%$ to $59 \%$. In [5], they combined eye-movement and EEG features to classify four emotions and obtained an average accuracy of $72.39 \%$. To the best of our knowledge, there has been no study on social emotion classification.

\subsection{Matrix-based classifier}

In conventional EEG classification, classifiers learn based on feature vectors, such as SVM [34] and Linear Discriminant Analysis (LDA) [35]. Therefore, features have to be stretched into a vector. This destroys the spatial relationship and results in the loss of discriminant information. Deep learning treats features as a form of matrix and has been widely used in diverse domains for classification. Convolutional Neural Network (CNN) is one of the prevalent deep learning models [36-38].

\section{Experiment setup}

The objective of our study aimed to investigate neural mechanisms of social emotion perception when two persons engaged in the same task. Since there was a potential cultural bias for Chinese participants using pictures from the International Affective Picture System (IAPS) [39], the native Chinese Affective Picture System (CAPS) has been used in the studies with Chinese participants [40,41]. Similarly, we used facial pictures from the native CAPS in this study.

Three hundred pictures were selected from the CAPS, which consisted of four emotion categories (anger 74, disgust 46, neutral 80, and happy 100). Each picture was presented to participants once at a random order. In the experiment, two participants watched the same pictures at the same time (see Fig. 2 for the experimental environment) and were asked to rate each picture in terms of valence and arousal at a scale from -5 to 5 through two paired iPad devices. One participant acted as a leader to first rate and the other participant acted as a follower to rate after his/her partner. The follower can see the rate made by the leader on the screen before he/she made the rate. Participants

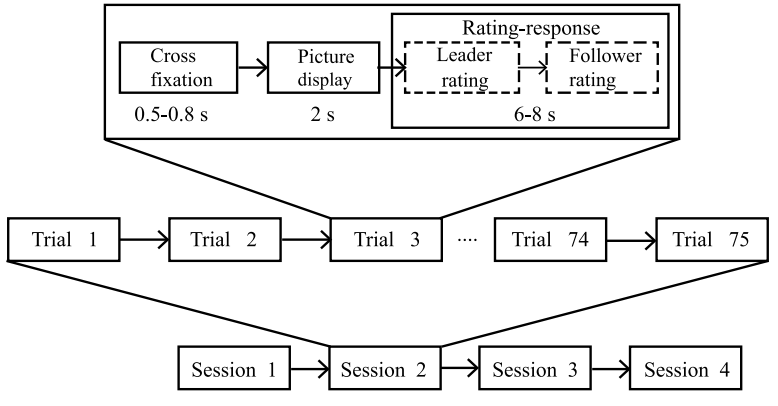

Fig. 3. Protocol of our designed experiment.

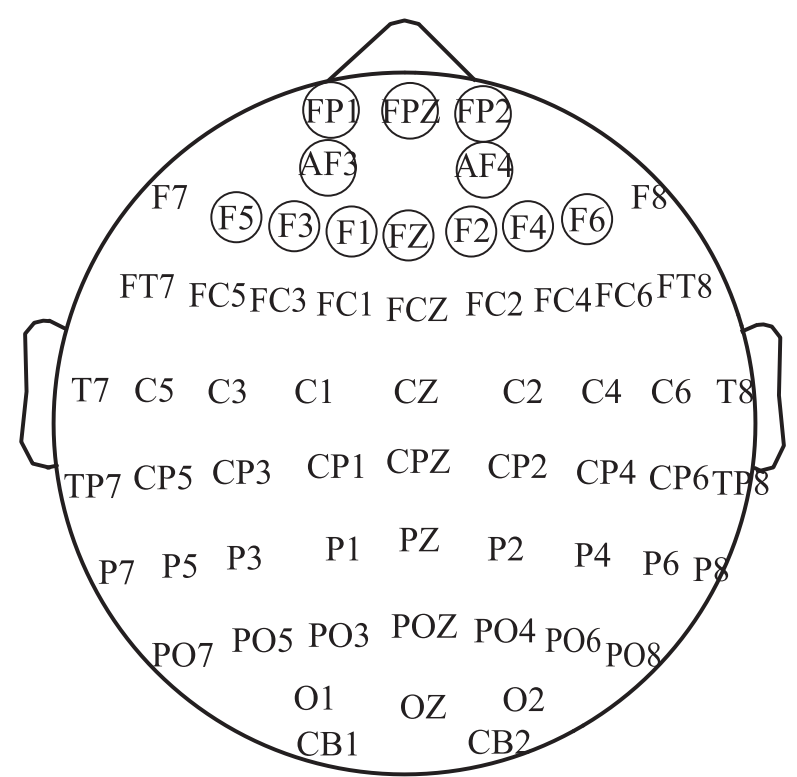

Fig. 4. The layout of 62 EEG electrodes. The selected electrodes used in this study were circled.

were instructed to rate based on how they really felt rather than the kind of emotion shown in pictures. There were four sessions in the experiment and each session consisted of 75 trials. Each trial lasted from $8.5 \mathrm{~s}$ to $10.8 \mathrm{~s}$, comprising picture stimulus for two seconds, prestimulus for a random time period varying from $500 \mathrm{~ms}$ to $800 \mathrm{~ms}$, and the rating-response period varying from 8 to $10 \mathrm{~s}$. The rating order was reversed in the last two sessions. Fig. 3 showed the protocol of our experiment. A short training phase was given to participants to familiarize them with the experiment before the formal sessions.

A total of 40 healthy, right handed participants (15 females) aged between 18 to 24 years old were divided into 20 pairs to perform the experiment. All subjects gave their written informed consents after the detailed introduction of the purpose and procedure of the experiment. EEG signals were acquired by two sets of amplifiers $(\mathrm{Ag} / \mathrm{AgCl}$, Biosemi, the Netherlands), each of them was with 62 electrodes placed according to the international 10-20 standards. These amplifiers were synchronized to record EEG at a sampling rate of $1000 \mathrm{~Hz}$. Based on the previous findings that the frontal region was dominantly related to emotion recognition and perception [42-44], we, therefore, selected twelve electrodes on this region in this study. The selected electrodes were FP1, FPZ, FP2, AF3, AF4, F5, F3, F1, FZ, F2, F4, and F6, which are shown in Fig. 4. 


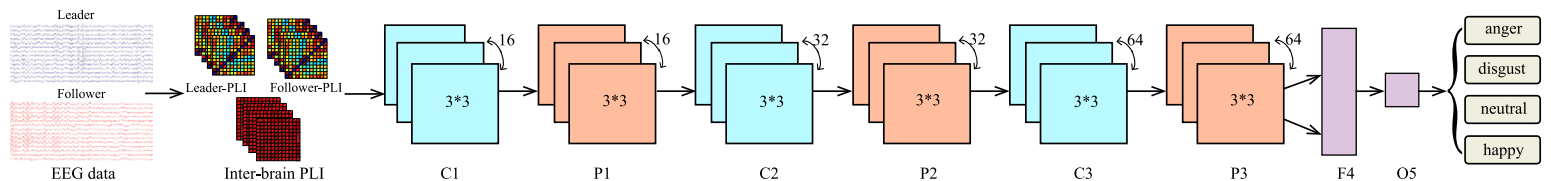

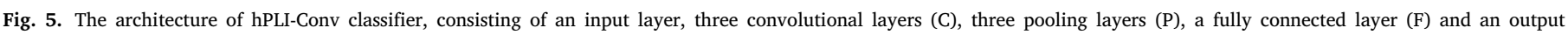
layer (O).

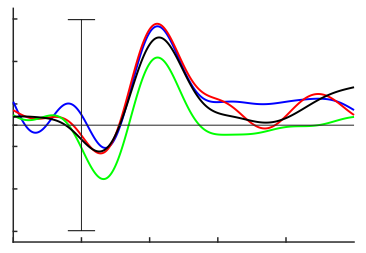

FP1*

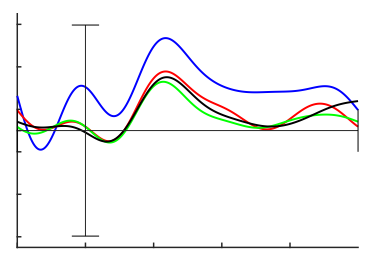

AF4*

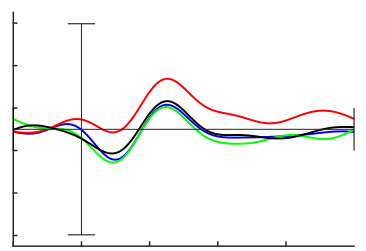

FZ*

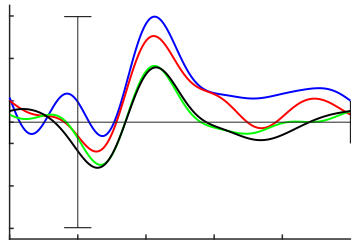

FPZ*

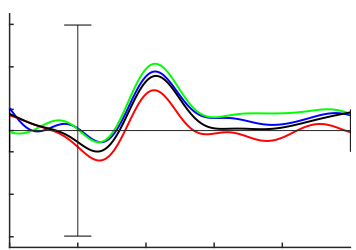

F5

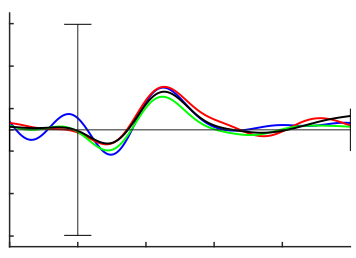

F2

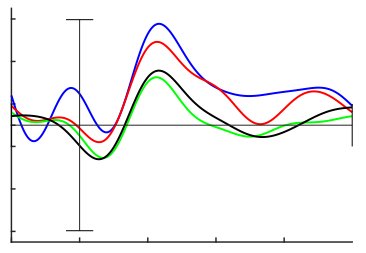

FP2*

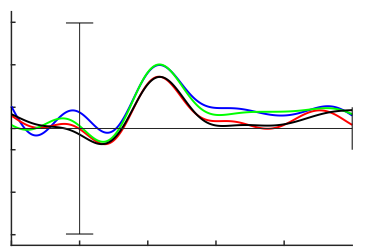

F3

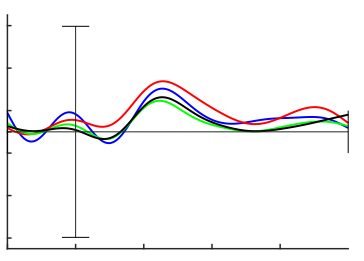

F4*

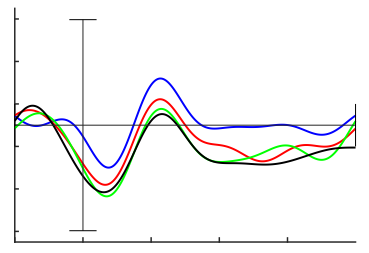

AF3*
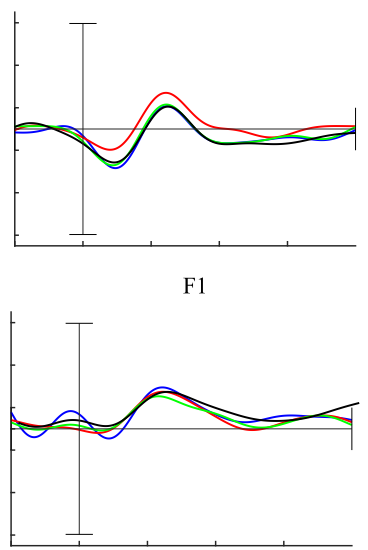

F6

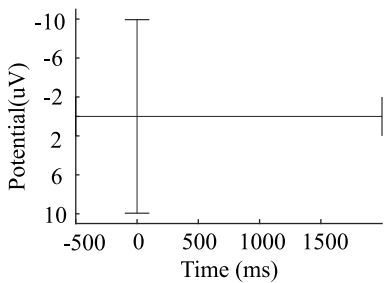

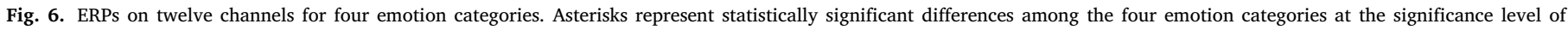

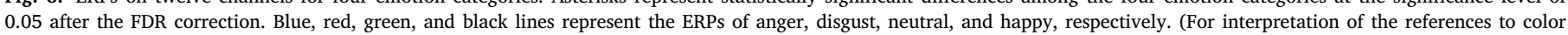
in this figure legend, the reader is referred to the web version of this article.)

\section{Methods}

\subsection{Preprocessing}

In the step of signal preprocessing, EEG data were band-pass filtered at the cut-off frequencies of $0.5 \mathrm{~Hz}$ and $85 \mathrm{~Hz}$, which was followed by common average reference (CAR) that the magnitude mean averaged across all electrodes was subtracted from the magnitudes of each electrode [45]. Independent Component Analysis (ICA) was subsequently used to obtain independent components, which was implemented in the EEGLAB [46]. The components representing artifacts were detected by using the MARA [47] (a plugin in the EEGLAB) and visual inspection. These artifact-related components were removed and the remaining components were used to reconstruct signals.

\subsection{Event related potential comparison}

Event-related potentials (ERPs) are particular bursts in magnitude, which are time-locked to specific events or stimuli [48]. This is a frequently-used marker to study brain activity. In this study, we explored ERPs for two cases (1) ERPs locked to stimuli, taking the epochs from $500 \mathrm{~ms}$ prior to the onsets of stimuli to the end of stimuli; (2) ERPs locked to rating responses, taking the epochs from $200 \mathrm{~ms}$ before to $800 \mathrm{~ms}$ after the rating responses of the paired participants. The epochs with obvious residual artifacts were excluded for further analysis. The epochs whose amplitudes exceeded $200 \mu \mathrm{V}$ or changes were greater than $100 \mu \mathrm{V}$ were also discarded [49]. The ERPs were obtained by averaging the remaining epochs, separately for stimuli and rating responses. 


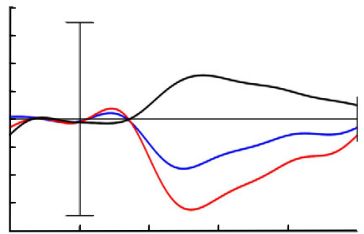

FP1

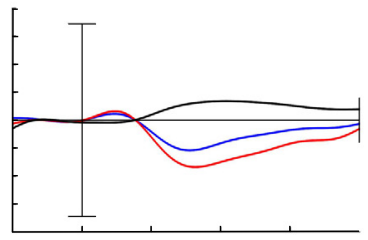

AF4

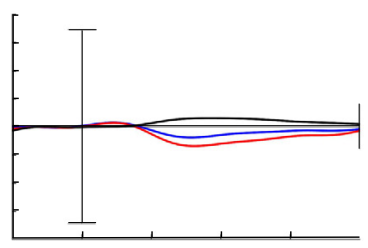

FZ

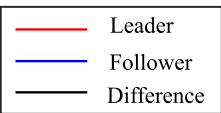

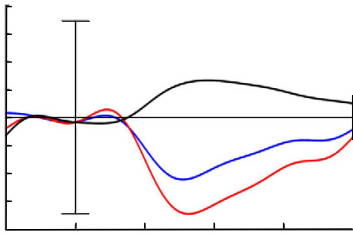

FPZ

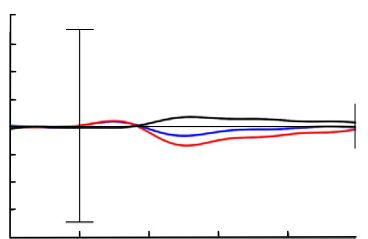

F5

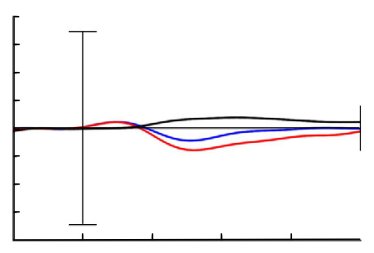

F2

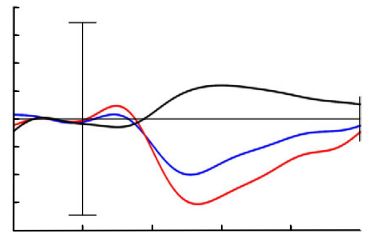

FP2

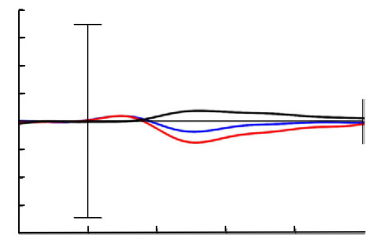

F3

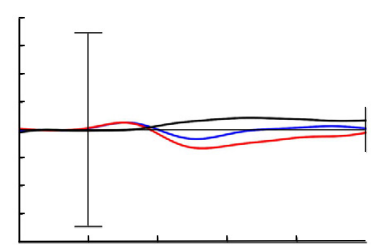

F4

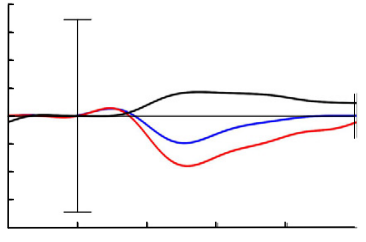

$\mathrm{AF} 3$

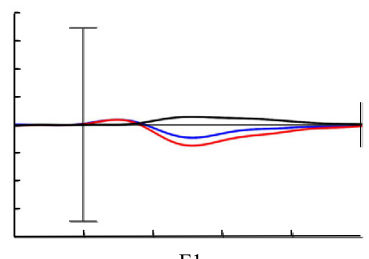

F1

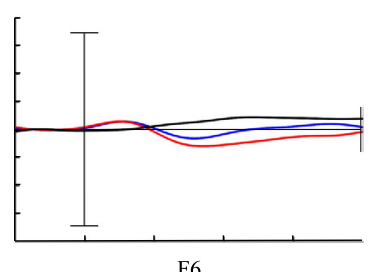

F6

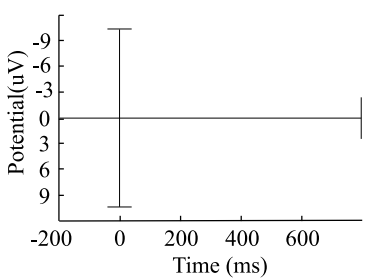

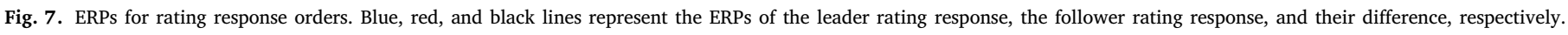

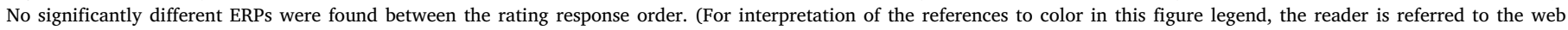
version of this article.)

ERPs among four emotion categories stimuli were compared and ERPs between the leader's responses and the follower's responses were compared. Specifically, for the comparison of stimuli, we made a matrix $a_{i j} \in \boldsymbol{A}_{40 \times 4}$ for each channel. Where $a_{i j}$ represents the mean averaged across 40 points around amplitude peak for the ith ( $i \in\{1,2, \ldots, 40\})$ participant's $j$ th $(j \in\{1,2,3,4\}$, which represent anger, disgust, neutral, and happy, respectively) stimulus category. For the comparison of responses, we compared the vector $\left\{E R P_{\text {leader }}(1), E R P_{\text {leader }}(2), \ldots, E R P_{\text {leader }}(40)\right\}$ with the vector $\left\{E R P_{\text {follower }}(1), E R P_{\text {follower }}(2), \ldots, E R P_{\text {follower }}(40)\right\}$, which were constructed from the average values of 40 points around the ERP peaks for each channel. One-way ANOVA was performed to evaluate whether or not there were significant differences in ERPs among four categories of stimulus and between rating responses (i.e., leader and follower rating responses). Additionally, in order to reduce the chance of type I error, false discovery rate (FDR) correction was applied to correct P-values.

\subsection{Intra- and inter-brain phase synchronization}

Phase lag index (PLI) was employed to estimate intra- and interbrain synchronizations, which was not sensitive to the volume conduction [50,52]. It can be calculated for each pair of channels by [51],

$P L I=|\langle\operatorname{sign}(\Delta \phi(t))\rangle|=\left|\frac{1}{T} \sum_{k=1}^{T} \operatorname{sign}\left(\Delta \phi\left(t_{k}\right)\right)\right|$

where $\mathrm{T}$ is the number of time points, $\Delta \phi^{\left(t_{k}\right)}$ is the instantaneous phase difference between two channels at time $t_{k}$. Its range is between 0 and
$1(0 \leq P L I \leq 1)$. A value of 0 indicates either no coupling or coupling with a phase difference centered around $0 \bmod \pi$. A value of 1 indicates perfect phase synchronization. It is worth pointing out that the phase difference is within the range of $-\pi<\Delta \phi<\pi$. If phase is defined using a range of $0<\Delta \phi<2 \pi$, then the formula (1) should be modified as

$$
P L I=\mid\langle\operatorname{sign}[\sin \Delta \phi(t)]\rangle
$$

In our case, 12 channels were selected, which resulted in a $12 \times 12$ matrix for each trial. Each entry in the matrix stood for synchronization value of a pair of channels. This synchronization calculation was done for all 20 pairs of participants, resulted in 20 inter-brain connectivity matrices and 40 intra-brain connectivity matrices. One-way ANOVA was applied to each entry (i.e., each connection) in the matrices to find out those which exhibited significant differences among four emotion categories. Again, FDR correction was used to control type I error.

\subsection{Classification}

CNN is one of the deep learning models and typically consists of convolution layer, pooling layer, and fully connected layer [36,53]. The convolution layer and pooling layer cooperate to form a stacked structure. Features were extracted and refined layer by layer and the prediction was given at the last layer. We constructed a CNN-based classifier for classifying hyper-scanning social emotions (Hereinafter referred to hPLI-Conv), which included intra-PLI-Conv (PLI features extracted from within-brain synchronization) and inter-PLI-Conv (PLI features extracted from the between-brain synchronization). The architecture of hPLI-Conv is shown in Fig. 5. 


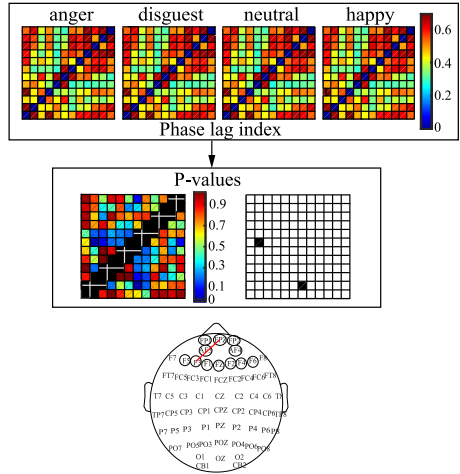

(A) Delta
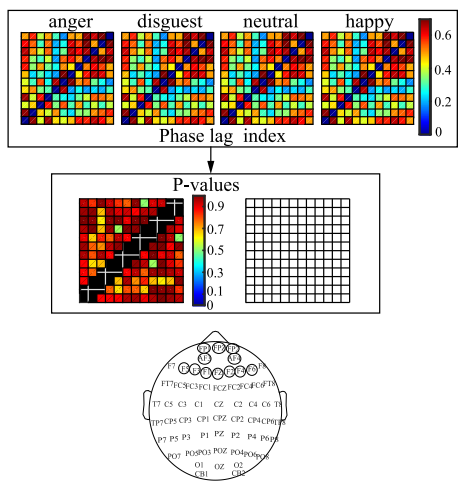

(D) Beta

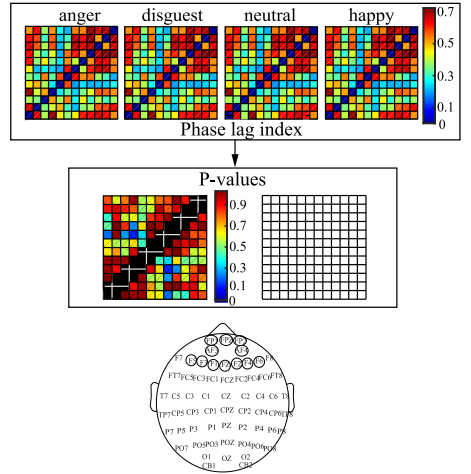

(B) Theta
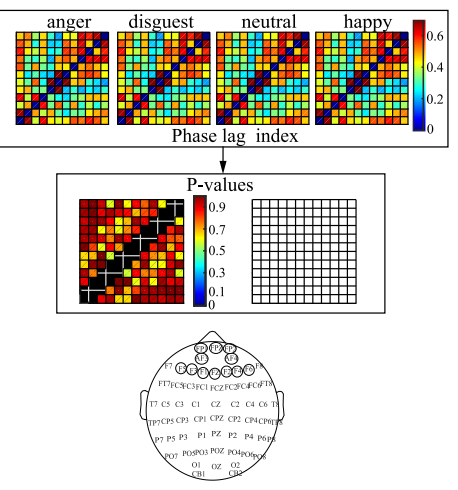

(E) Gamma

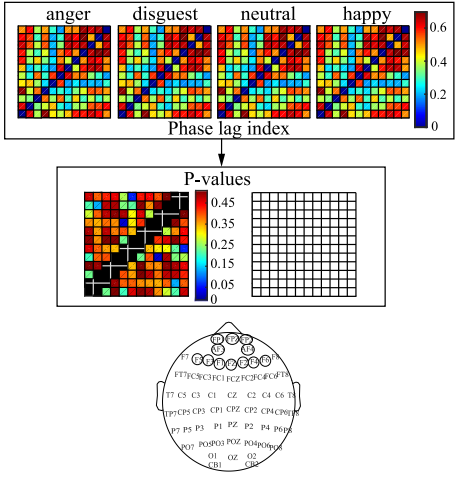

(C) Alpha

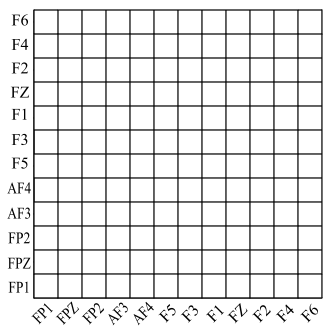

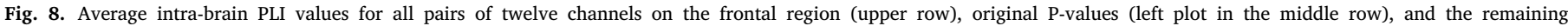
connections which were still significantly different after the multiple comparison correction (right plot in the middle row).

The intra-brain PLI (PLI extracted from leader or follower in the same paired group) or inter-brain PLI matrix of $12 \times 12$ was input to the first convolutional layer ( $C 1$ in Fig. 5$)$ and was convolved with a kernel with the size of $3 \times 3$. This layer was followed by a pooling (maxpooling) layer ( $P 1$ in Fig. 5). The convolution and pooling were alternated in the following layers until the fully connected layer (see $F 4$ in Fig. 5). In addition, dropout strategy was used to avoid overfitting issue (dropout ratio $=0.2$ ). The final softmax layer (see $O 5$ in Fig. 5) converted features into probabilities for each class, computing as

$$
\begin{array}{r}
P\left(Y=i \mid O_{h}, W_{o}, B_{o}\right) \mid=\operatorname{softmax}\left(W_{o} O_{h}+B_{o}\right) \\
=\frac{e^{W_{o i} O_{h}+B_{o i}}}{\sum_{j}^{e_{o j} O_{h}+B_{o j}}}
\end{array}
$$

where $W_{o}$ is a weight matrix, $B_{o}$ is a bias vector and $O_{h}$ is the output of $O 5$. The rectified linear unit (ReLU) activation function was used in the convolutional layers $(C 1, C 2, C 3)$ and fully connected layer $F 4$, which was defined as

$\operatorname{Re} L U(x)=\max (0, x)$

ReLU is the max function between 0 and input $x$. The strides of each layer was set as 1 and the size of kernel is $3 \times 3$.

\section{Results}

\subsection{Event related potential}

Fig. 6 shows the ERPs of four emotion categories for the selected twelve channels located on frontal region. It can be seen that the ERPs for anger on the channels FP1, FPZ, FP2, AF3, and AF4 and for disgust on the channels F1, FZ and F4 were larger than the ERPs for happy on those channels. Statistical results were listed in Table 1. P-values are
Table 1

One-way ANOVA results for ERP comparisons among four emotion categories.

\begin{tabular}{lll}
\hline Channel & P-value & Q-value \\
\hline FP1 & 0.0034 & 0.0136 \\
FPZ & 0.0009 & 0.0103 \\
FP2 & 0.0230 & 0.0394 \\
AF3 & 0.0031 & 0.0186 \\
AF4 & 0.0045 & 0.0108 \\
F5 & 0.0388 & 0.0582 \\
F3 & 0.1664 & 0.1664 \\
F1 & 0.0474 & 0.0569 \\
FZ & 0.0039 & 0.0117 \\
F2 & 0.0417 & 0.0556 \\
F4 & 0.0046 & 0.0092 \\
F6 & 0.0500 & 0.0545 \\
\hline
\end{tabular}

Table 2

Average accuracies (\%) of inter-PLI-Conv (using inter-brain PLI features) and intra-PLI-Conv (using intra-brain PLI features) for all five typical frequency bands.

\begin{tabular}{lllllll}
\hline Feature & & $\delta$ & $\theta$ & $\alpha$ & $\beta$ & $\gamma$ \\
\hline \multirow{2}{*}{ Inter } & Mean & 61.46 & 62.25 & $\mathbf{6 5 . 5 5}$ & 60.05 & 55.42 \\
& Std & 0.35 & 0.41 & 0.42 & 0.34 & 0.34 \\
\hline \multirow{2}{*}{ Intra } & Mean & 50.06 & 48.96 & $\mathbf{5 0 . 7 7}$ & 47.71 & 47.46 \\
& Std & 0.59 & 0.48 & 0.40 & 0.36 & 0.35 \\
\hline
\end{tabular}

original significance level while Q-values are significance level after multiple comparison correction (using FDR). There were statistically significant differences in the ERPs on the channels of FP1, FPZ, FP2, AF3, AF4, FZ and F4.

For the ERP comparisons of response order, we found that the ERP magnitude of the leader's rating response was less than that of the 


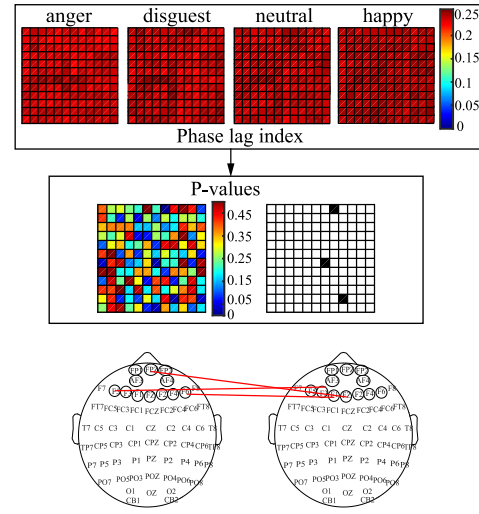

(A) Delta
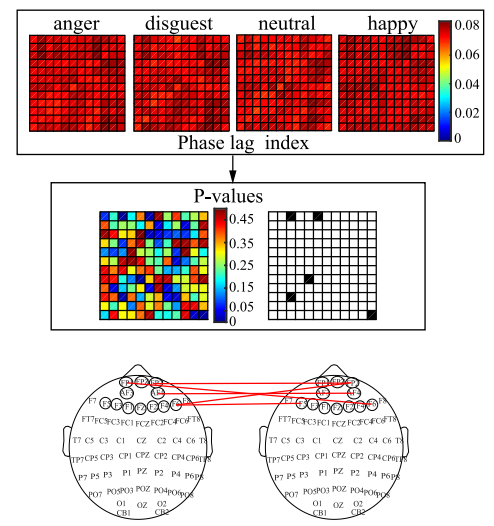

(D) Beta
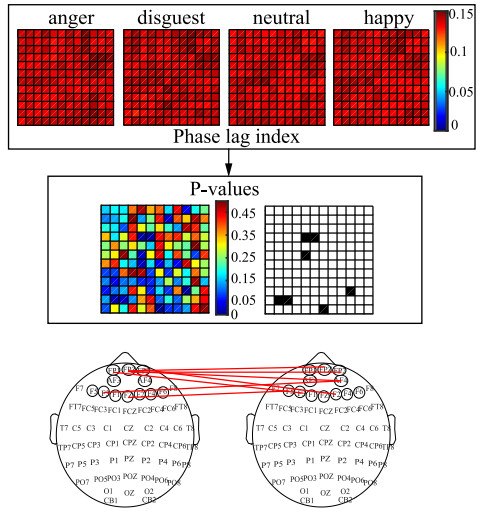

(B) Theta
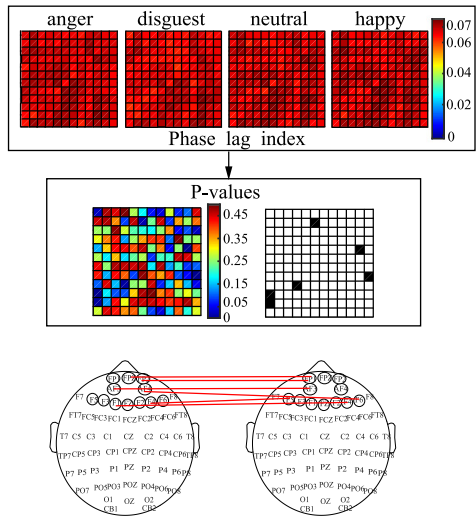

(E) Gamma
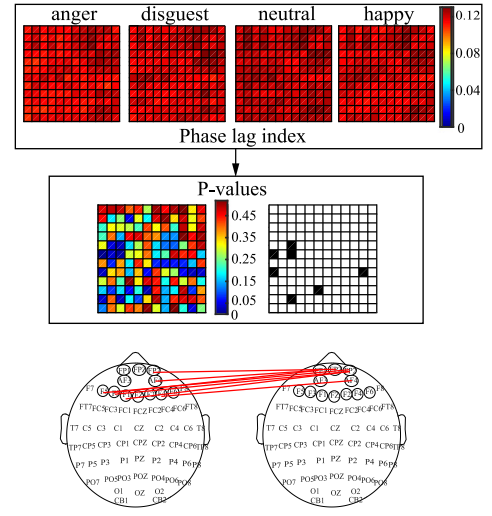

(C) Alpha

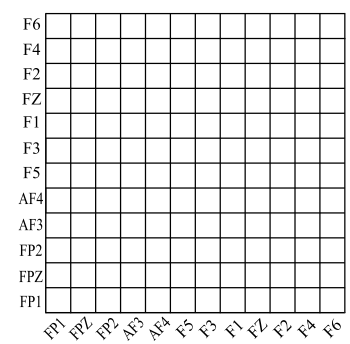

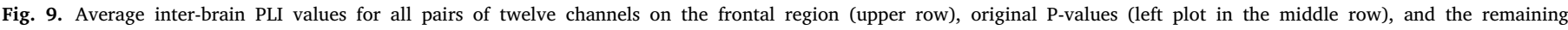
connections which were still significantly different after the multiple comparison correction (right plot in the middle row).

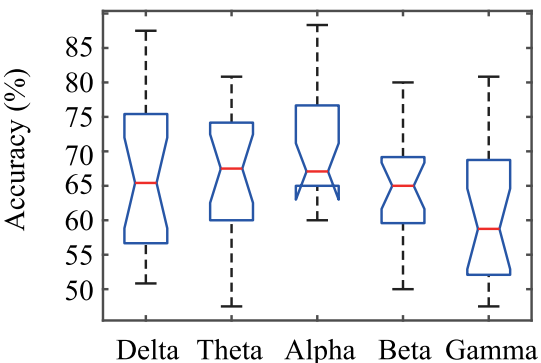

(A) Inter-PLI-Conv

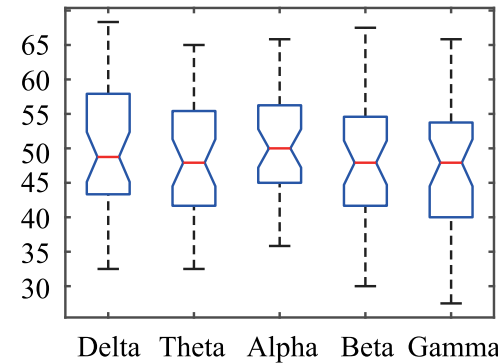

(B) Intra-PLI-Conv

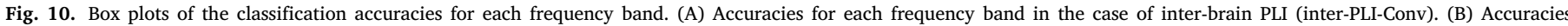
for each frequency band in the case of intra-brain PLI (intra-PLI-Conv).

follower's rating response for all channels (see Fig. 7). However, the differences were not significant $(p>0.05)$ for all channels.

\subsection{Intra- and inter-brain phase synchronization}

We explored average intra-brain synchronizations among four emotion categories as detailed in Section 4.3. The results for all five typical frequency bands were shown in Fig. 8. In each subplot in Fig. 8, the matrix shown in the top row was average PLI values for all pairs of twelve channels on the frontal region. The left matrix in the middle row shows original $\mathrm{P}$-values and the right matrix in the middle row shows the remaining connections which were still significant after the multiple comparison correction. The bottom topography illustrates the significant connections after the multiple comparison correction if any. Only one intra-brain connection (i.e., connection from FPZ to F3) in delta band was retained after the multiple comparison correction (see Fig. 8(A)). In general, the connective strengths of the channels located on the right frontal region were higher than that of the left frontal region.

We also explored inter-brain synchronization in the same way and showed the results in Fig. 9. We found much more significantly different connections after the multiple comparison correction in this case of inter-brain synchronization, appearing in all five typical frequency bands. It is worth noting that the inter-brain connective strengths were generally much smaller than that of the intra-brain connective strengths according to the results. 


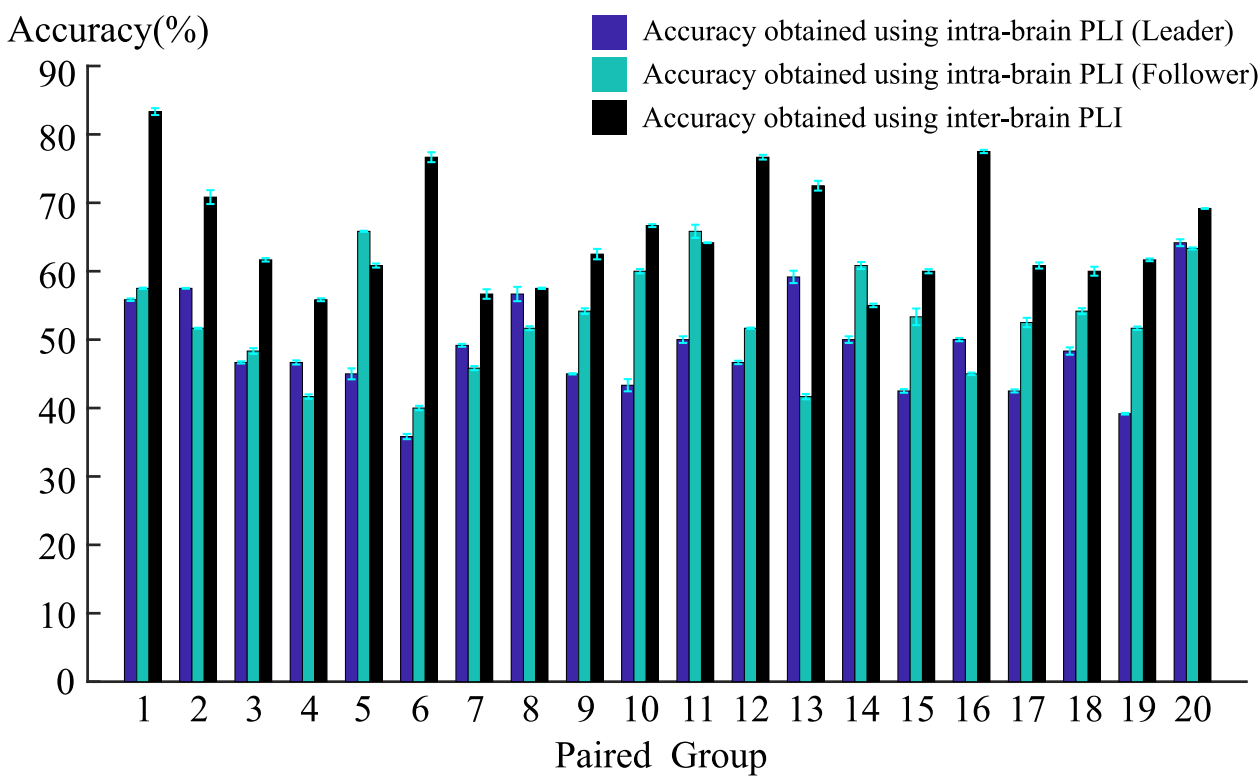

Fig. 11. Accuracies of intra-PLI-Conv (using intra-brain PLI) and inter-PLI-Conv (using inter-brain PLI) for each pair of participants.

\begin{tabular}{c|c|c|c|c|}
\multicolumn{4}{c}{ Anger } & \multicolumn{2}{c}{ Disgust } & Neutral \\
\cline { 3 - 5 } Anger & 49.0 & 14.1 & 15.2 & 21.7 \\
\cline { 2 - 5 } Disgust & 32.5 & 30.0 & 16.2 & 21.3 \\
\cline { 2 - 5 } Neutral & 14.0 & 7.0 & 60.4 & 18.6 \\
\cline { 2 - 5 } & 10.3 & 8.0 & 14.7 & 67.0 \\
\cline { 2 - 5 } Happy & 10.0 &
\end{tabular}

(A) Inter-PLI-Conv

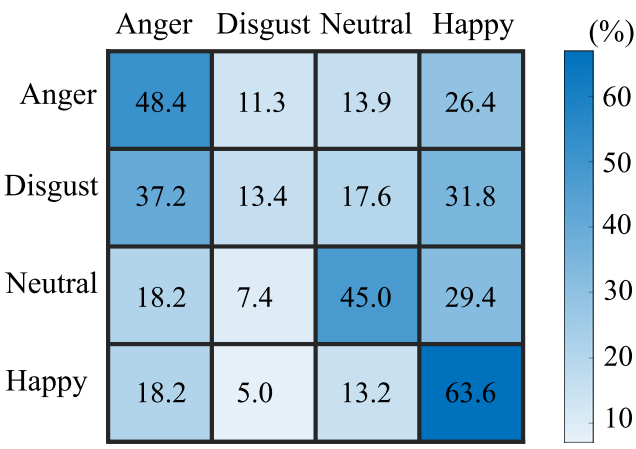

(B) Intra-PLI-Conv

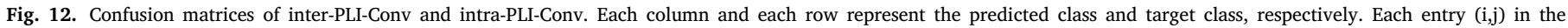
matrix shows the percentage of the samples which belong to class i but are misclassified as class j. (A) Inter-PLI-Conv (B) Intra-PLI-Conv.

\subsection{Classification}

\subsubsection{Analysis of different bands}

We evaluated classification performance in terms of accuracy for all five frequency bands using the channels located on the frontal region. Our objective was to investigate how the performance varied when different frequency bands and different synchronizations (intra-brain PLI and inter-brain PLI) were used. The classification performance was evaluated by five-fold cross-validation ( $80 \%$ samples including all four categories were for the training and the remaining $20 \%$ samples were for the testing in each time of the total of five times). The average accuracies and standard deviations for each frequency band are summarized in Table 2. From the table, we can see that the inter-PLI-Conv outperformed the intra-PLI-Conv, showing the higher accuracies for all frequency bands and the lower standard deviations for all frequency bands except the alpha band. One-way ANOVA showed that there was significant difference in classification accuracy among frequency bands in the case of inter-brain PLI $(p<0.01)$ but no significant difference in the case of intra-brain PLI $(p>0.05)$. The performance was best in the alpha band in the case of inter-brain PLI (see Fig. 10).

\subsubsection{EEG-based emotion recognition}

Based on the above results, we selected alpha band to evaluate classification performance of EEG-based emotion recognition. As shown in Fig. 11, the inter-PLI-Conv (obtained from inter-brain PLI) outperformed the intra-PLI-Conv (obtained from intra-brain PLI) for all paired groups except for the paired group 5, 11 and 14. Although the follower with accuracy of $65.83 \%$ is higher than inter-PLI-Conv with accuracy of $60.83 \%$ in paired group 5 and follower with accuracy of $65.81 \%$ is higher than inter-PLI-Conv with accuracy of $64.17 \%$ in paired group 11 , the paired participants averaging accuracy of intra-PLI-Conv in paired group 5 is $57.92 \%$ and $57.91 \%$ in paired group 11 , which are still lower than inter-PLI-Conv accuracy.

To further investigate the classification performance for each emotion category, we showed the confusion matrices, which reveal how many samples were misclassified to another class (see Fig. 12). We observed that inter-PLI-Conv had an advantage of classifying neutral $(60.4 \%)$ and happy $(67.0 \%)$ emotion categories compared to the intra-PLI-Conv (45.0\% for neutral and $63.6 \%$ for happy).

\section{Conclusion}

In this paper, we explored social emotion perception and emotion classification with the channels located on the frontal region. We focus on frontal lobe because (1) it was reported that the frontal lobe plays an important role in emotion perception; (2) from the perspective of practical application of emotion recognition, less number of channels 
could reduce computational complexity and facilitate the setup. In this study, we investigated social emotion from the aspects (1) time-locked mechanisms and the influence caused by the social interactions; (2) synchronization comparisons between intra-brain and inter-brain under the identical experimental setting; (3) classification performances using intra-brain PLI and inter-brain PLI.

We proposed an EEG-based hyper-scanning social emotion perception experiment. According to the ERP analyses, we discovered that there were significant differences among four emotion categories (i.e., anger, disgust, neutral, and happy) on the explored channels located on the frontal region and there was no significant difference between rating orders. From the results of PLI, we observed that (1) the values of intra-brain PLI are greater than that of inter-brain PLI across four emotions and five frequency bands; (2) the number of significantly different inter-brain PLI connections is more than that of intra-brain PLI. We designed a CNN-based classifier for social emotion classification and compared performances when using different kinds of features. Results show that the classification performance of interPLI-Conv outperforms that of intra-PLI-Conv for all frequency bands. It shows that the best performance was achieved when using alpha frequency band compared to other four bands.

Our study demonstrated that the better classification performance was obtained when the inter-brain PLI was used as features compared to the intra-brain PLI. Previous studies have shown that social interaction promotes the synchronization between brains [16,54-56], when multiple subjects engaged in a collaborative task. Moreover, another study found that performance could be enhanced in the context of multi-user brain computer interface compared to the case of single user [57]. This performance enhancement was also found in the cooperative working mode [58] and larger group for decision making [59]. Alpha band was relevant to the social interactions [13,55,60] and affectively emotional stimuli [61,62], which might explain why the highest classification performance was obtained when using features in alpha band.

\section{Declaration of competing interest}

The authors declare that they have no known competing financial interests or personal relationships that could have appeared to influence the work reported in this paper.

\section{Acknowledgments}

The work presented in this paper was supported by the National Natural Science Foundation of China (No. 61633010, 61806149, 61673322), and the National Key Basic Research Program of China (No. 2013CB329502). This research was also partially supported by the Ministry of Education and Science of the Russian Federation (grant 14.756.31.0001) and the Polish National Science Center (grant 2016/20/W/N24/00354).

\section{References}

[1] M. Pantic, L.J. Rothkrantz, Toward an affect-sensitive multimodal humancomputer interaction, Proc. IEEE 91 (9) (2003) 1370-1390.

[2] W.L. Parry-Jones, Historical aspects of mood and its disorders in young people, in: I. Goodyer (Ed.), The Depressed Child and Adolescent, 2001, pp. 1-23.

[3] A. Etkin, T.D. Wager, Functional neuroimaging of anxiety: a meta-analysis of emotional processing in PTSD, social anxiety disorder, and specific phobia, Am. J. Psychiatry 164 (10) (2007) 1476-1488.

[4] I.B. Mauss, M.D. Robinson, Measures of emotion: A review, Cogn. Emot. 23 (2) (2009) 209-237.

[5] W.-L. Zheng, W. Liu, Y. Lu, B.-L. Lu, A. Cichocki, EmotionMeter: A multimodal framework for recognizing human emotions, IEEE Trans. Cybern. 49 (3) (2018) 1110-1122.

[6] M.L. Phillips, W.C. Drevets, S.L. Rauch, R. Lane, Neurobiology of emotion perception I: the neural basis of normal emotion perception, Biol. Psychiatry 54 (5) (2003) 504-514.

[7] C.G. Kohler, J.B. Walker, E.A. Martin, K.M. Healey, P.J. Moberg, Facial emotion perception in schizophrenia: a meta-analytic review, Schizophr. Bull. 36 (5) (2009) 1009-1019.
[8] P.M. Niedenthal, L.W. Barsalou, P. Winkielman, S. Krauth-Gruber, F. Ric, Embodiment in attitudes, social perception, and emotion, Pers. Soc. Psychol. Rev. 9 (3) (2005) 184-211.

[9] R. Cowie, E. Douglas-Cowie, N. Tsapatsoulis, G. Votsis, S. Kollias, W. Fellenz, J.G. Taylor, Emotion recognition in human-computer interaction, IEEE Signal Process. Mag. 18 (1) (2001) 32-80.

[10] K. Löwith, L. Feuerbach und der Ausgang der klassischen deutschen Philosophie, Riv. Filos. 17 (1928) 323.

[11] S.E. Barkan, Sociology: Understanding and changing the social world, Flat World Knowledge, Incorporated, 2011.

[12] G.A. Van Kleef, How emotions regulate social life: The emotions as social information (EASI) model, Curr. Dir. Psychol. Sci. 18 (3) (2009) 184-188.

[13] S. Dikker, L. Wan, I. Davidesco, L. Kaggen, M. Oostrik, J. McClintock, J. Rowland, G. Michalareas, J.J. Van Bavel, M. Ding, et al., Brain-to-brain synchrony tracks real-world dynamic group interactions in the classroom, Curr. Biol. 27 (9) (2017) 1375-1380.

[14] S. Cutini, S.B. Moro, S. Bisconti, Functional near infrared optical imaging in cognitive neuroscience: an introductory review, J. Near Infrared Spectrosc. 20 (1) (2012) 75-92.

[15] P.R. Montague, G.S. Berns, J.D. Cohen, S.M. McClure, G. Pagnoni, M. Dhamala, M.C. Wiest, I. Karpov, R.D. King, N. Apple, et al., Hyperscanning: Simultaneous fMRI During Linked Social Interactions, Academic Press, 2002.

[16] N. Sinha, T. Maszczyk, Z. Wanxuan, J. Tan, J. Dauwels, EEG hyperscanning study of inter-brain synchrony during cooperative and competitive interaction, in: 2016 IEEE International Conference on Systems, Man, and Cybernetics, SMC, IEEE, 2016, pp. 004813-004818.

[17] K. Watanabe, M. Kashino, K. Nakazawa, S. Shimojo, Implicit ambient surface information: From personal to interpersonal, in: IUI Workshops, 2018, pp. 1-5.

[18] T.R. Powell, S. De Jong, G. Breen, C.M. Lewis, D. Dima, Telomere length as a predictor of emotional processing in the brain, Hum. Brain Mapp. (2018).

[19] A.R. Vaidya, L.K. Fellows, Ventromedial frontal lobe damage affects interpretation, not exploration, of emotional facial expressions, Cortex (2019).

[20] J. Drapeau, N. Gosselin, I. Peretz, M. McKerral, Emotional recognition from dynamic facial, vocal and musical expressions following traumatic brain injury, Brain Inj. 31 (2) (2017) 221-229.

[21] X. Cui, D.M. Bryant, A.L. Reiss, NIRS-based hyperscanning reveals increased interpersonal coherence in superior frontal cortex during cooperation, Neuroimage 59 (3) (2012) 2430-2437.

[22] F. Babiloni, F. Cincotti, D. Mattia, F.D.V. Fallani, A. Tocci, L. Bianchi, S. Salinari, M. Marciani, A. Colosimo, L. Astolfi, High resolution EEG hyperscanning during a card game, in: Engineering in Medicine and Biology Society, 2007. EMBS 2007. 29th Annual International Conference of the IEEE, IEEE, 2007, pp. 4957-4960.

[23] A. Flexer, S. Makeig, Independent component analysis of EEG recorded during two-person game playing, Appl. Artif. Intell. 21 (9) (2007) 883-894.

[24] T. Liu, H. Saito, M. Oi, Role of the right inferior frontal gyrus in turn-based cooperation and competition: a near-infrared spectroscopy study, Brain Cogn. 99 (2015) 17-23.

[25] J. Li, Y. Liu, Z. Lu, L. Zhang, A competitive brain computer interface: Multiperson car racing system, in: 2013 35th Annual International Conference of the IEEE Engineering in Medicine and Biology Society, EMBC, IEEE, 2013, pp. 2200-2203.

[26] L. Astolfi, F. De Vico Fallani, J. Toppi, F. Cincotti, S. Salinari, G. Vecchiato, C. Wilke, H. Yuan, B. He, F. Babiloni, Imaging the social brain by simultaneous hyperscanning of different subjects during their mutual interactions, IEEE Intell. Syst. 26 (2011) 38-45.

27] K. Spiegelhalder, S. Ohlendorf, W. Regen, B. Feige, L.T. van Elst, C. Weiller, J. Hennig, M. Berger, O. Tüscher, Interindividual synchronization of brain activity during live verbal communication, Behav. Brain Res. 258 (2014) 75-79.

[28] F. Babiloni, L. Astolfi, Social neuroscience and hyperscanning techniques: past, present and future, Neurosci. Biobehav. Rev. 44 (2014) 76-93.

[29] K. Yun, D. Chung, J. Jeong, Emotional interactions in human decision making using EEG hyperscanning, in: International Conference of Cognitive Science, 2008, pp. 1-4.

[30] G. Dumas, F. Lachat, J. Martinerie, J. Nadel, N. George, From social behaviour to brain synchronization: review and perspectives in hyperscanning, Irbm 32 (1) (2011) 48-53.

[31] M. Li, B.-L. Lu, Emotion classification based on gamma-band EEG, in: Engineering in Medicine and Biology Society, 2009. EMBC 2009. Annual International Conference of the IEEE, IEEE, 2009, pp. 1223-1226.

[32] B. Kaur, D. Singh, P.P. Roy, EEG based emotion classification mechanism in BCI, Procedia Comput. Sci. 132 (2018) 752-758.

[33] Z. Lan, O. Sourina, L. Wang, R. Scherer, G. Müller-Putz, Unsupervised feature learning for EEG-based emotion recognition, in: 2017 International Conference on Cyberworlds, CW, IEEE, 2017, pp. 182-185.

[34] D. Garrett, D.A. Peterson, C.W. Anderson, M.H. Thaut, Comparison of linear, nonlinear, and feature selection methods for EEG signal classification, IEEE Trans. Neural Syst. Rehabil. Eng. 11 (2) (2003) 141-144.

[35] A. Subasi, M.I. Gursoy, EEG signal classification using PCA, ICA, LDA and support vector machines, Expert Syst. Appl. 37 (12) (2010) 8659-8666.

[36] H. Zeng, C. Yang, G. Dai, F. Qin, J. Zhang, W. Kong, EEG classification of driver mental states by deep learning, Cogn. Neurodyn. 12 (6) (2018) 597-606. 
[37] Y.R. Tabar, U. Halici, A novel deep learning approach for classification of EEG motor imagery signals, J. Neural Eng. 14 (1) (2016) 016003.

[38] U.R. Acharya, S.L. Oh, Y. Hagiwara, J.H. Tan, H. Adeli, D.P. Subha, Automated EEG-based screening of depression using deep convolutional neural network, Comput. Methods Programs Biomed. 161 (2018) 103-113.

[39] H. Yuxia, LUO Yuejia Key Laboratory of Mental Health, Institute of Psychology, Chinese Academy of Sciences, Beijing 100101; Native assessment of international affective picture system, Chin. Mental Health J. 9 (2004)

[40] X. Meng, J. Yuan, H. Li, Automatic processing of valence differences in emotionally negative stimuli: evidence from an ERP study, Neurosci. Lett. 464 (3) (2009) 228-232.

[41] J. Yuan, J. Zhang, X. Zhou, J. Yang, X. Meng, Q. Zhang, H. Li, Neural mechanisms underlying the higher levels of subjective well-being in extraverts: Pleasant bias and unpleasant resistance, Cogn. Affect. Behav. Neurosci. 12 (1) (2012) 175-192.

[42] A.S. Heberlein, A.A. Padon, S.J. Gillihan, M.J. Farah, L.K. Fellows, Ventromedial frontal lobe plays a critical role in facial emotion recognition, J. Cogn. Neurosci. 20 (4) (2008) 721-733

[43] Y.-P. Lin, C.-H. Wang, T.-P. Jung, T.-L. Wu, S.-K. Jeng, J.-R. Duann, J.-H. Chen, EEG-based emotion recognition in music listening, IEEE Trans. Biomed. Eng. 57 (7) (2010) 1798-1806.

[44] W.-L. Zheng, B.-L. Lu, Investigating critical frequency bands and channels for EEG-based emotion recognition with deep neural networks, IEEE Trans. Autonom. Mental Dev. 7 (3) (2015) 162-175.

[45] H. Ramoser, J. Muller-Gerking, G. Pfurtscheller, Optimal spatial filtering of single trial EEG during imagined hand movement, IEEE Trans. Rehabil. Eng. 8 (4) (2000) 441-446.

[46] A. Delorme, S. Makeig, EEGLAB: an open source toolbox for analysis of singletrial EEG dynamics including independent component analysis, J. Neurosci. Methods 134 (1) (2004) 9-21.

[47] I. Winkler, S. Brandl, F. Horn, E. Waldburger, C. Allefeld, M. Tangermann, Robust artifactual independent component classification for BCI practitioners, J. Neural Eng. 11 (3) (2014) 035013.

[48] T. Picton, S. Bentin, P. Berg, E. Donchin, S. Hillyard, R. Johnson, G. Miller, W. Ritter, D. Ruchkin, M. Rugg, et al., Guidelines for using human eventrelated potentials to study cognition: recording standards and publication criteria, Psychophysiology 37 (2) (2000) 127-152.

[49] S.S. Kang, D.P. Dionisio, S.R. Sponheim, Abnormal mechanisms of antisaccade generation in schizophrenia patients and unaffected biological relatives of schizophrenia patients, Psychophysiology 48 (3) (2011) 350-361.
[50] C.J. Stam, G. Nolte, A. Daffertshofer, Phase lag index: assessment of functional connectivity from multi channel EEG and MEG with diminished bias from common sources, Hum. Brain Mapp. 28 (11) (2007) 1178-1193.

[52] M.F. PY, T. Hashimoto, G. Li, J. Okuda, T. Konno, K. Samejima, J. Morita, Phase synchrony in symbolic communication: Effect of order of messaging bearing intention, in: Proceedins of the 20th Annual International Conference of the Japanese Society for Language Sceiences, 2018, pp. 40-41.

[51] S. Kasakawa, T. Yamanishi, T. Takahashi, K. Ueno, M. Kikuchi, H. Nishimura, Approaches of phase lag index to EEG signals in Alzheimer disease from complex network analysis, in: Innovation in Medicine and Healthcare 2015, Springer, 2016, pp. 459-468.

[53] U.R. Acharya, S.L. Oh, Y. Hagiwara, J.H. Tan, H. Adeli, Deep convolutional neural network for the automated detection and diagnosis of seizure using EEG signals, Comput. Biol. Med. 100 (2018) 270-278.

[54] G. Dumas, J. Nadel, R. Soussignan, J. Martinerie, L. Garnero, Inter-brain synchronization during social interaction, PLoS One 5 (8) (2010) e12166.

[55] A. Bezerianos, Y. Sun, Y. Chen, K.F. Woong, F. Taya, P. Arico, G. Borghini, F. Babiloni, N. Thakor, Cooperation driven coherence: Brains working hard together, in: 2015 37th Annual International Conference of the IEEE Engineering in Medicine and Biology Society, EMBC, IEEE, 2015, pp. 4696-4699.

[56] U. Lindenberger, S.-C. Li, W. Gruber, V. Müller, Brains swinging in concert: cortical phase synchronization while playing guitar, BMC Neurosci. 10 (1) (2009) 22.

[57] D. Valeriani, A. Matran-Fernandez, Past and future of multi-mind brain-computer interfaces, in: Brain-Computer Interfaces Handbook: Technological Theoretical Advances, CRC Press, 2018, pp. 685-700.

[58] R. Poli, C. Cinel, A. Matran-Fernandez, F. Sepulveda, A. Stoica, Towards cooperative brain-computer interfaces for space navigation, in: Proceedings of the 2013 International Conference on Intelligent User Interfaces, ACM, 2013, pp. $149-160$.

[59] N.L. Kerr, R.S. Tindale, Group performance and decision making, Annu. Rev. Psychol. 55 (2004) 623-655.

[60] V. Müller, U. Lindenberger, Hyper-brain networks support romantic kissing in humans, PLoS One 9 (11) (2014) e112080.

[61] I.H. Gotlib, EEG alpha asymmetry, depression, and cognitive functioning, Cogn. Emot. 12 (3) (1998) 449-478.

[62] M. Balconi, G. Mazza, Lateralisation effect in comprehension of emotional facial expression: a comparison between EEG alpha band power and behavioural inhibition (BIS) and activation (BAS) systems, Laterality: Asymmetries Body Brain Cogn. 15 (3) (2010) 361-384. 